www.nature.com/ejhg

\title{
ARTICLE
}

\section{Clinical variability in calpainopathy: What makes the difference?}

\author{
Flávia de Paula ${ }^{1}$, Mariz Vainzof ${ }^{1}$, Maria Rita Passos-Bueno ${ }^{1}$, Rita de Cássia M Pavanello ${ }^{1}$,
} Sergio Russo Matioli ${ }^{1}$, Louise VB Anderson ${ }^{3}$, Vincenzo Nigro ${ }^{2}$ and Mayana Zatz ${ }^{*, 1}$

${ }^{1}$ Human Genome Research Center-Departamento de Biologia, IB Universidade de São Paulo, Brazil; ${ }^{2}$ TIGEM and Dipartimento di Patologia Generale, Seconda Universita'degli studi di Napoli, Italy; ${ }^{3}$ Neurobiology Department, University Medical School, Framlington Place, Newcastle upon Tyne, UK

Limb girdle muscular dystrophies (LGMD) are a heterogeneous group of genetic disorders characterised by progressive weakness of the pelvic and shoulder girdle muscles and a great variability in clinical course. LGMD2A, the most prevalent form of LGMD, is caused by mutations in the calpain-3 gene (CAPN3). More than 100 pathogenic mutations have been identified to date, however few genotype : phenotype correlation studies, including both DNA and protein analysis, have been reported. In this study we screened 26 unrelated LGMD2A Brazilian families (75 patients) through Single-Stranded Conformation Polymorphism (SSCP), Denaturing high-performance liquid chromatography (DHPLC) and sequencing of abnormal fragments which allowed the identification of $\mathbf{4 7}$ mutated alleles (approximately 90\%). We identified two recurrent mutations (R110X and 2362-2363AG > TCATCT) and seven novel pathogenic mutations. Interestingly, 41 of the identified mutations (approximately $80 \%$ ) were concentrated in only 6 exons $(1,2,4,5,11$ and 22), which has important implications for diagnostic purposes. Protein analysis, performed in 28 patients from 25 unrelated families showed that with exception of one patient (with normal/slight borderline reduction of calpain) all others had total or partial calpain deficiency. The effects of type of mutation, amount of calpain in the muscle, gender and ethnicity of affected patients on clinical course (age of onset and ascertainment) were analysed. Interestingly, it was observed that, on average, African-Brazilian calpainopathy patients are more severely affected than Caucasians.

European Journal of Human Genetics (2002) 10, 825 - 832. doi:10.1038/sj.ejhg.5200888

Keywords: LGMD2A; calpainopathy; calpain-3; limb-girdle muscular dystrophy type 2A; screening of mutation; genotype $\times$ phenotype correlation; clinical variability

Introduction

Limb girdle muscular dystrophies (LGMD) include a heterogeneous group of disorders characterised by progressive weakness of the pelvic and shoulder girdle muscles and a highly variable clinical course. Ten autosomal recessive (LGMD2) and six autosomal dominant (LGMD1) forms have been related to date. ${ }^{1-3}$ Linkage analysis indicates that

\footnotetext{
*Correspondence: M Zatz, Human Genome Research Center, Departamento de Biologia - IBUSP, Universidade de São Paulo, Rua do Matão 277 sala 211 Cidade Universitária; São Paulo SP CEP 05508-900, Brasil; Tel: 55 (11) 3091-7563; 55 (11) 3091-7581; Fax: 55 (11) 3091-7419; E-mail: mayazatz@ib.usp.br or flapvit@yahoo.com.br Received 28 February 2002; revised 30 July 2002; accepted 1 August 2002
}

there are other genes causing both $\mathrm{AR}$ as well as $\mathrm{AD}$ LGMD. ${ }^{4-8}$

LGMD2A is the most prevalent form of LGMD in the European as well as in the Brazilian population. ${ }^{1,9}$ It is caused by mutations in the gene that encodes the proteolytic enzyme calpain-3 mapped at 15q15.1-q21.1. ${ }^{10}$ The human CAPN-3 gene comprises 24 exons and covers a genomic region of $50 \mathrm{~kb}$. It is expressed as a $3.5 \mathrm{~kb}$ transcript, and translated a $94 \mathrm{kD}$ protein. Calpain-3 is a skeletal muscle-specific member of the calpain superfamily, which process various intracellular kinases, phosphatases, phospholipases, transcription factors and cytoskeletal proteins to modulate their activities. Although the 
functional role of calpain-3 is still under discussion, it has been suggested that it may play a significant role in intracellular signal transduction systems. ${ }^{11-14}$ According to Baghdiguian et al., ${ }^{15}$ calpain-3 deficiency would cause myonuclear apoptosis and a profound perturbation of the $\mathrm{I} \kappa \mathrm{B} \alpha / \mathrm{NF}-\kappa \mathrm{B}$ pathway.

Ninety-seven pathogenic mutations, spread over almost all exons of the CAPN-3 gene have been identified in a large multicenter study performed in a Caucasian population. ${ }^{16}$ Most of them (70\%) represent private variants although particular mutations were found more frequently in some populations. In addition six other mutations were identified exclusively in LGMD2A patients from Japan. ${ }^{17}$ However, few genotype phenotype studies, including both DNA as well as protein analysis have been reported to date. Explaining the great inter and intrafamilial clinical variability observed in affected patients remains a great challenge.

In the present study, we screened the CAPN-3 gene in 26 unrelated Brazilian LGMD2A families through SSCP, DHPLC and sequencing of abnormal fragments. This led to the identification of two recurrent mutations, six preferentially mutated exons and the characterisation of seven novel pathogenic mutations. Western blot (WB) analysis of calpain-3 performed on muscle biopsies from patients from 25 unrelated families showed that, except for one patient, who showed a normal/borderline amount of calpain on muscle, all other had total or partial calpain deficiency.

The effect of the type of mutation, amount of calpain in muscle, gender and ethnicity of affected patients on two clinical parameters (age of onset and ascertainment) were analysed.

\section{Subjects and methods \\ Subject}

A total of 26 unrelated Brazilian families (including 75 affected patients) referred to the Center for the Study of the Human Genome Study, at the Department of Biology, University of São Paulo, Brazil, were included in the present investigation. All studies were performed following patients' informed consent.

The diagnosis of LGMD was based on clinical and neurological examination, family history, muscle biopsy studies, elevated serum creatine-kinase levels and electromyography, according to the criteria reported in Bushby and Beckmann. ${ }^{18}$

The families were classified in LGMD type 2A through linkage analysis (eight families) or calpain deficiency on muscle but in whom seven other muscle protein (dystrophin, $\alpha, \beta, \gamma$ and $\delta$ sarcoglycan, dysferlin and telethonin) showed normal results (17 families). One patient (family \#15) who showed discordant results in two independent calpain WB analysis (a slight reduction in the first but an apparently normal amount in the second) and normal results for the other seven proteins was also included.
The Brazilian population is highly miscigenated. Therefore, in order to compare patients from different ethnic background they were classified as Caucasians (C) or African/Brazilian (A/B) according to the criteria published in Azevedo. ${ }^{19}$ No patient in the present sample was Oriental (although in São Paulo there are 1000000 Brazilian/Japanese).

\section{Method}

Protein Western blot analysis Muscle calpain-3 analysis was performed in a total of 28 patients, from 25 unrelated families. In one family (\#25) no muscle sample was available for protein analysis. Muscle samples were obtained from biceps biopsies, frozen in liquid nitrogen immediately after removal and stored at $-70^{\circ} \mathrm{C}$ until use. The $94 \mathrm{kD}$ calpain-3 band was analysed using the monoclonal Calp3C/12A2 antibody. ${ }^{20}$ The multiplex Western blot methodology for studying simultaneously calpain, dystrophin, and dysferlin proteins was performed as described previously. $^{21,22}$

DNA analysis Genomic DNA was extracted from peripheral blood as described in Miller et al. ${ }^{23}$ The DNA was amplified by PCR with the primers published by Richards et $a l .^{24}$ The screening of the Calpain-3 gene was done through Single Strand Conformation Polymorphism (SSCP) and Denaturing High Performance Liquid Chromatography (DHPLC) for all 24 exons of the gene. Samples with abnormal migration on SSCP gel or with heteroduplex profile on DHPLC analysis were sequenced in an automatic sequencing machine (ABI Model 377 Version 3.0).

In order to verify whether recurrent mutations in unrelated families had a common origin, highly polymorphic markers - D15S779, D15S782, D15S778, D15S783, distance less than $1 \mathrm{~cm}(3-4 \mathrm{Mb})$ from the CAPN-3 gene ${ }^{25}$ were used, according to standard procedures. ${ }^{26}$ Haplotypes were constructed and compared between families.

Statistical analysis The comparisons of continuous variables among groups were done with the ANOVA test with the Welch correction for heterocedasticity. ${ }^{27}$ To detect possible effects on clinical heterogeneity, we used the Tukey-Kramer HSD test. For the statistical analysis the level of significance was considered as 0.05. The statistical analyses were carried out with the JMP program (1994, SAS Institute, NC, USA).

\section{Results}

Mutations analysis

Forty-seven of the 52 alleles (approximately 90\%) had their mutation identified. However only 18 distinct mutations were found: 11 missense pathogenic changes, six null mutation and one splice site alteration. Two recurrent mutations were found in 12 unrelated families: 
(i) 2362-2363AG > TCATCT in exon 22 (highly prevalent in Basque patients ${ }^{28}$ was found in 12 alleles from seven families (12/52 or approximately $20 \%$ ): five in homozygozity and two in compound heterozygotes (in one the other mutation was not found).

(ii) R110X in exon 2 was present in eight alleles from five families (8/52 or approximately 15\%): three in homozygozity and two in compound heterozygotes (R110X/R572W and R110X/R748Q).

Haplotype analysis using four microsatellites (D15S779, D15S782, D15S778, D15S783) flanking the LGMD2A locus confirmed that affected patients carrying the R110X as well as the 2362AG > TCATCT mutation shared each one the same haplotypes: ( $\left.\begin{array}{llll}7 & 6 & 4 & 5\end{array}\right)$ and (1 $\left.\begin{array}{llll}1 & 2 & 4 & 3\end{array}\right)$ respectively, suggesting a common origin for these two mutations.

Another interesting result is that among the 47 detected mutations, $41(87 \%)$ were concentrated in only six exons: $1,2,4,5,11$ and 22 that altogether encode 39\% of the protein. The distribution of mutations in the 26 families, patients' mean age at onset and ascertainment and the results of muscle calpain-3 analysis are summarised in Table 1.

Seven novel mutations: three missense, two frameshift, one nonsense and one in frame deletion were identified (Table 2). All three missense changes (K211E, P82L,
M248R) were not found in 100 chromosomes from Brazilian controls.

Patients from the 26 families were screened for all calpain-3 gene exons. But in five of them the second mutation was not identified due to the limited sensibility of the methods, or because the change lies in the non-coding region of the gene.

\section{Polymorphic changes}

Four polymorphic changes, all previously reported by other groups (www.dmd.nl) were found: two silence changes, T96C (exon 1) and T606C (exon 4) which code for proline and serine, respectively, were found in two patients from two unrelated families (\#5 and \#15) and in 3.5\% of the controls, always in heterozygosity. One patient (\#5) carries the 2380+12delA change (exon 22) in heterozygous state. The most frequent polymorphic change, the G706A change (exon 5) with an allelic frequency of $18 \%$ in the normal Brazilian controls, was found in five patients, two in homozygous state, family (\#4) and three in heterozygosity (family \#3).

\section{Clinical findings and calpain-3 deficiency}

A similar pattern of muscle involvement but with different rate of progression was observed with proximal weakness

Table 1 Results of DNA analysis, presence of consanguinity (C), number and gender of affected patients per family, age at onset and ascertainment (Asc) of the propositus, CK fold increase, the ethnicity (Eth.) and results of muscle calpain analysis in the 26 LGMD2A Brazilian families

\begin{tabular}{|c|c|c|c|c|c|c|c|c|c|c|c|}
\hline$F$ & $\begin{array}{l}\text { Pathogenic } \\
\text { changes }\end{array}$ & State & Exon & Consequence & C & $\begin{array}{l}\text { No. aff } \\
\text { gender }\end{array}$ & $\begin{array}{l}\text { Age at } \\
\text { onset }\end{array}$ & Asc. & $C K X \uparrow$ & Eth. & $\begin{array}{l}\text { Calpain } \\
\text { deficiency }\end{array}$ \\
\hline 1 & P82L & Hom & 1 & Missense & Y & $1 \mathrm{~F}$ & 18 & 28 & 14 & $\mathrm{C}$ & Partial \\
\hline 2 & P82L/G496R & Comp het & $1 / 11$ & Missense & $\mathrm{N}$ & $2 \mathrm{~F}$ & $32.5 \pm 0.7$ & 36 & $23 \pm 1.4$ & $\mathrm{C}$ & Partial \\
\hline 3 & $\mathrm{R} 748 \mathrm{X} / \mathrm{P} 82 \mathrm{~L}$ & Comp het & $1 / 21$ & nonsense/missense & $N$ & $1 \mathrm{~F}: 2 \mathrm{M}$ & $10.6 \pm 0.5$ & 16 & $27 . \overline{3} \pm 7.5$ & C & Partial \\
\hline 4 & $260^{\wedge} 265$ delTCTTTTinsGA & Hom & 1 & Frameshift & $N$ & $2 \mathrm{~F}$ & 10 & 16 & $26 \pm 19.8$ & - & Partial \\
\hline 5 & $60 \operatorname{del} \mathrm{A} / ?$ & Het/? & $1 / ?$ & Frameshift/? & $N$ & $2 \mathrm{~F}: 2 \mathrm{M}$ & $23 \pm 7.7$ & 39 & $7.5 \pm 4.0$ & $\mathrm{~A} / \mathrm{B}$ & Partial \\
\hline 6 & R110X & Hom & 2 & Nonsense & $\mathrm{N}$ & $2 \mathrm{~F}: 2 \mathrm{M}$ & $14^{-}$ & 15 & $29 . \overline{3} \pm 27$ & $\mathrm{~A} / \mathrm{B}$ & Total \\
\hline 7 & R110X & Hom & 2 & nonsense & $\mathrm{Y}$ & $5 \mathrm{~F}: 6 \mathrm{M}$ & $8.75 \pm 1.9$ & 33 & $19.3 \pm 15$ & $\mathrm{~A} / \mathrm{B}$ & Total \\
\hline 8 & R110X & Hom & 2 & nonsense & $Y$ & $2 \mathrm{M}$ & $9-$ & 19 & $23.0 \pm 9.8$ & $\mathrm{C}$ & Total \\
\hline 9 & R110X/R572W & Comp het & $2 / 13$ & nonsense/missense & $N$ & $2 \mathrm{~F}: 1 \mathrm{M}$ & $12.5 \pm 0.7$ & 25 & $9.3 \pm \overline{4} .1$ & C & Total \\
\hline 10 & $\mathrm{R} 110 \mathrm{X} / \mathrm{R} 748 \mathrm{Q}$ & Comp het & $2 / 21$ & nonsense/missense & $N$ & $3 M$ & $13 \pm \overline{1} .4$ & 17 & $42 \pm 15.8$ & $A / B$ & Total \\
\hline 11 & $509-510$ insA & Hom & 4 & frameshift & Y & $2 \mathrm{~F}$ & $12 . \overline{5} \pm 2.1$ & 20 & $6.4 \pm 4.6$ & C & Total \\
\hline 12 & $\mathrm{~K} 211 \mathrm{E} / ?$ & Het/? & $4 / ?$ & Missense/? & $N$ & $1 F$ & 18 & 25 & 30 & $\mathrm{~A} / \mathrm{B}$ & Total \\
\hline 13 & $\mathrm{~K} 211 \mathrm{E} / ?$ & Het/? & $4 / ?$ & Missense/? & - & $1 \mathrm{~F}$ & - & - & - & - & Partial \\
\hline 14 & M248R & Hom & 5 & Missense & $N$ & $2 \mathrm{~F}: 2 \mathrm{M}$ & 13 & 30 & $7.5 \pm 5$ & C & Partial \\
\hline 15 & $254 \Delta \mathrm{K} / \mathrm{R} 490 \mathrm{~W}$ & Comp het & $5 / 11$ & $\begin{array}{l}\text { In frame deletion/ } \\
\text { missense }\end{array}$ & $\mathrm{N}$ & $1 \mathrm{M}$ & 18 & 25 & 12 & C & $\begin{array}{l}\text { Partial/ } \\
\text { normal }\end{array}$ \\
\hline 16 & R490W/V354G & Comp het & $8 / 11$ & Missense & $\mathrm{N}$ & $5 \mathrm{M}$ & $15 \pm 2.1$ & 14 & $24.5 \pm 33$ & C & Partial \\
\hline 17 & $1194-9 A>G / 1194-9 A>G$ & Hom & 10 & Splicing & Y & $1 F$ & 14 & 25 & 8.6 & C & Partial \\
\hline 18 & $2362 \mathrm{AG} \rightarrow \mathrm{TCATCT}$ & Hom & 22 & Frameshift & Y & $7 F: 6 \mathrm{M}$ & 13 & 29 & $10.1 \pm 14$ & C & Partial \\
\hline 19 & 2362AG $\rightarrow$ TCATCT & Hom & 22 & Frameshift & $N$ & $1 F$ & 15 & 20 & 2 & C & Total \\
\hline 20 & 2362AG $\rightarrow$ TCATCT & Hom & 22 & Frameshift & Y & $1 F$ & 11 & 11 & 34 & C & Total \\
\hline 21 & 2362AG $\rightarrow$ TCATCT & Hom & 22 & Frameshift & $N$ & $1 \mathrm{M}$ & 2 & 10 & 12 & C & Total \\
\hline 22 & $2362 \mathrm{AG} \rightarrow \mathrm{TCATCT}$ & Hom & 22 & Frameshift & $\mathrm{N}$ & $2 \mathrm{M}$ & $12.5 \pm 0.7$ & 20 & $62 \pm 76$ & $A / B$ & Total \\
\hline 23 & $\begin{array}{l}2362 \mathrm{AG} \rightarrow \mathrm{TCATCT} / \\
254 \Delta \mathrm{K}\end{array}$ & Comp het & $22 / 5$ & $\begin{array}{l}\text { Frameshift/ } \\
\text { In frame deletion }\end{array}$ & $N$ & $1 \mathrm{M}$ & $10-$ & 16 & $54^{-}$ & C & Partial \\
\hline 24 & 2362AG $\rightarrow$ TCATCT/? & Het/? & $22 / ?$ & Frameshift/? & $N$ & $1 \mathrm{~F}$ & 7 & 20 & 6 & C & Total \\
\hline 25 & R769Q & Hom & 22 & Missense & Y & $4 \mathrm{~F}: 1 \mathrm{M}$ & 16 & 26 & $5.6 \pm 5.8$ & C & - \\
\hline 26 & $765 \Delta \mathrm{l} / ?$ & Het/? & $22 / ?$ & In frame deletion/? & $\mathrm{N}$ & $1 \mathrm{~F}$ & 45 & 52 & 15 & C & Partial \\
\hline
\end{tabular}

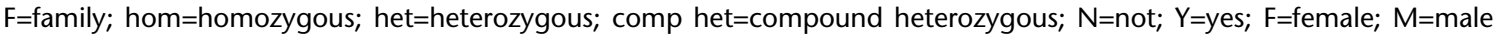


Table 2 Novel mutation: characterization of seven novel mutations in Brazilian LGMD2A patients

\begin{tabular}{lllll}
\hline Exon & DNA mutation & Amino acid change & Consequence & Restriction enzyme \\
\hline 1 & C245T & P82L & Missense & Mspl \\
1 & $260^{\wedge} 265$ delTCTTTinsGA & - & Frameshift & - \\
4 & A631G & K211E & Missense & - \\
4 & $509^{\wedge} 510$ ins & Y170X & Nonsense & - \\
5 & T743G & M248R & Missense & AfllII \\
21 & C2242T & R748X & Nonsense & Sfanl \\
22 & $2295^{\wedge} 2297$ delTCA & 765 dell & In frame deletion & - \\
\hline
\end{tabular}

starting in the lower limbs and progressing to the upper limbs shortly after. All patients lost the ability to walk on heels before the capacity to walk on toes. Calve's hypertrophy was observed in $75 \%$ of the patients.

Since two large groups of patients carried the same null mutations, most of them in homozygosity, the clinical course was compared among them. In 20 patients carrying the 2362-2363AG > TCATCT mutation, the average age at onset and the progression of the disease was highly variable despite the fact that 18 of them were homozygous for this mutation. In five patients from four families (\#19, 20 and 22 homozygous and 23, a compound heterozygous) the mean age at onset was $12.2 \pm 1.9$ (from 10 to 15 years old). Patients from the remaining three families (\#18 and 21 , homozygous and \#24, compound heterozygous) showed a more severe course. In families 24 and 21 where the probands were isolated cases the age at onset was 7 and 2 years, respectively (they are currently, 27 and 13). In the first consanguineous family (\#18) with 13 patients, three affected brothers had a severe Duchenne-like course and were confined to a wheelchair at age 13, while other affected sisters or cousins are only mildly affected in their twenties.

Twenty-two patients carried the R110X stop codon mutation. In four of these families (\#6 and 8 homozygous; 9 and 10 compound heterozygous both for missense changes) the average age at onset was $12.5 \pm 1.8$ (from 9 to 14 years old). However in one large homozygous consanguineous family (\#7) the clinical course was highly variable: one affected male patient was unable to walk at age 19 years old while other affected relatives were mildly affected in their thirties.

This great inter and intra family variability has already been described not only in LGMD2A patients ${ }^{16}$ but also in LGMD patients in general. ${ }^{1,2,5}$ Here we confirmed this observation in a large calpainopathy sample including a total of 42 patients that carry the same recurrent mutations.

\section{Muscle calpain-3}

Muscle calpain was completely absent in 12 patients from 12 families and partially deficient in 12 families. Total calpain deficiency was observed in: (a) the five families with the R110X stop codon mutation (3 homozygous, \#6, 7, 8 and 2 compound heterozygotes, \#9 and 10); (b) in five of the seven families with the frameshift $2362 \mathrm{AG} \rightarrow$ TCATCT mutation (4 homozygote \#19, 20, 21 and 22 and 1 on just one allele, \#24); (c) in one family with a missense mutation detected on just one allele (\#12) and (d) in one homozygous for a stop codon mutation (\#11).

Partial calpain deficiency was observed: (a) in four families with missense mutation (\#1,2, 14, and 16), (b) in two families with frameshift mutations (\#4 and 18), (c) in one patient with splicing mutation (\#17), (d) in two families compound heterozygotes for missense/nonsense changes $(\# 3,23),(e)$ and in three families in which only one mutation was identified (families \#5, 13 and 26, one frameshift change, one missense mutation and one in frame deletion respectively).

One patient (family \#15), a compound heterozygote for one missense and one 'in frame' deletion showed a normal/ borderline amount in two independent WB analysis.

\section{Consanguinity and ethnic background}

Eight among the 25 families (32\%) were consanguineous, 17 were non-consanguineous $(68 \%)$ and in one (\#13) no reliable information could be obtained.

The ethnic background assessed in 24 families, showed that 18 were Caucasians (C) (included 48 patients) and six families African-Brazilian (A/B) (24 affected persons).

\section{Discussion}

\section{Calpain mutations}

In most populations, calpainopathy has been shown to be the most prevalent form of LGMD. In Brazil, it corresponds to about $30 \%$ of the known AR-LGMD. ${ }^{1}$ In the present study the observation that most of the ascertained families are non consanguineous (68\%) and Caucasian (75\%) is in accordance with the relatively high frequency of calpain mutations in the Caucasian population. More than 100 pathogenic mutations may cause LGMD2A, including the seven novel mutations reported here. Most of the previously reported one $(70 \%)$ represent private variants, although some mutations were found more frequently in isolated communities, with a high degree of inbreeding. ${ }^{16,28,29}$ In a recent study of 21 Japanese LGMD2A patients, 10 of 13 identified mutations were not found in other populations (including six novel ones). Three of them, accounting for $71 \%$ of the cases, were recurrent mutations. ${ }^{17}$ 
Although the Brazilian population is highly miscigenated we found two recurrent mutations present in about $46 \%$ of our LGMD families: R110X and 2362-2363EG> TCATCT. Haplotype analysis suggest a common origin for both mutations, suggesting a founder effect. The 23622363AG $>$ TCATCT mutation is a frequent change found in Basque communities $^{28}$ while the R110X change, previously detected only in Brazilian patients ${ }^{16,24}$ was recently identified in three Italian patients (Ms in preparation).

Other rare mutations, previously described in patients from European countries or North America included: R572W (exon 13), R748Q (exon 21) and 60delA (exon 1) in Spain; V354G (exon 8) and $254 \Delta \mathrm{K}$ (exon 5) in France; G496R (exon 11) in Italy and R490W (exon 11) in North America. ${ }^{16,24,29}$ The R769Q (exon 22) mutation, frequent in the Amish community ${ }^{24,25}$ was present in one consanguineous Brazilian family.

One intronic homozygous change, 1194-9A $>$ G, was detected in an isolated 25-year-old female, with a mild course (onset at age 14) born from consanguineous parents (family \#17). This change, which affects splicing has been already reported in two French patients in heterozygozity (www.dmd.nl). Although this mutation could represent a polymorphism the fact that it was associated with muscle calpain deficiency and was absent in 100 normal Brazilian controls suggests that it is a pathogenic change. In addition this same change in homozygosity was recently reported by Chae et al. ${ }^{17}$ in Japanese patients.

\section{Mutation distribution}

Previously reported Calpain-3 mutations showed that they are distributed along the 24 exons, with a slight mutational 'hot spot' in exon 21. ${ }^{16}$ Two mutations in this exon were found in the present study. One is a rare novel mutation: R748X, which results from a change CGA $>$ TGA in the nucleotide position 2242, creating a stop codon. The second missense R748Q change (position 2243: CGA>CAA) was previously described in European patients. ${ }^{16,29,30}$

In the present sample, exons 1 and 22 concentrated more distinct mutations. Three independent mutations were found in exon 1, two of them novel changes: $260^{\wedge} 265$ delTCTTTTinsGA a frameshift mutation in a consanguineous genealogy with two affected sibs and the P82L missense change found in six patients from three families. The 60delA change, present in one genealogy with four patients was reported previously in the Spanish population. ${ }^{16}$ In exon 22, in addition to the recurrent Basque mutation 2362-2363AG>TCATCT, two other mutations were identified: one novel (765dell) and one previously reported (R769Q) frequently found in the Amish community.

The distribution of mutations along the gene in this Brazilian sample showed that 41 of the 47 identified mutations were concentrated in only six exons. This finding indicates that, for diagnostic purposes, the screening of six exons: 1, 2, 4, 5, 11 and 22 would allow the identification of $80 \%$ of the mutated alleles. Since the frequency of LGMD2A in Brazilian patients was estimated as $30 \%{ }^{1,6}$ the probability to find a mutation in a LGMD patient prior to muscle protein analysis is about $24 \%$. This observation may be particular helpful for patients who are severely affected and in whom getting a muscle biopsy may be difficult or for patients who show normal results for all known LGMD proteins including calpain-3 amount. ${ }^{20}$

\section{Type of mutation versus amount of muscle calpain-3} Correlation analysis between the type of mutation and calpain amount was done for 20 families in whom both mutations were identified. In 10 families with no detectable calpain, eight were homozygous for two null mutations (nonsense or frameshift) while two were compound heterozygotes for one nonsense and one missense mutation. In nine families with partial calpain deficiency, four had missense mutations on both alleles, one was homozygous for a splicing mutation, two were homozygous for two frameshift mutations and two were compound heterozygotes (one in frame deletion/frameshift mutation and one missense/frameshift mutation). The patient with normal/ borderline calpain amount was a compound heterozygote.

Therefore, although null mutations were more often associated with total absence of calpain while missense mutation with partial deficiency, no direct correlation between the type of mutation and calpain amount was seen. This occurred in particular because a total absence or only a reduction in calpain-3 amount was seen in patients homozygous for the same frameshift mutation (families \#18 and 19). Finally, the apparently normal calpain-3 amount in patient from family \#15 is in accordance with previous studies. ${ }^{20}$ Although the age of onset in this patient occurred at age 18, his disease is progressing rapidly (at age 25 he needs help to raise up from a chair) suggesting that although present in muscle the protein is probably not functional.

\section{Clinical course according to the nature of mutation}

For analysis of the clinical course two parameters were considered: age at ascertainment and age at onset. Age at ascertainment is directly related to the severity of the phenotype since patients who are more affected look for medical help earlier than those who have a milder course.

Although there is a marked inter and intrafamilial heterogeneity in the severity of the clinical course in LGMD2A muscular dystrophy ${ }^{1,2,6,16}$ it has been suggested that on average missense mutations are usually associated to a milder phenotype than null mutations. ${ }^{16,17,20}$

In the present sample, patients from all families in whom both mutations were identified, were classified in three groups according to the nature of the mutation: 18 patients (nine males and nine females) had two missense mutations 
in both alleles or one missense and one in frame deletion (group 1); 10 patients (seven males and three females) were compound heterozygous for missense/null mutations (group 2) and 40 patients (19 males and 21 females) had null mutations (frameshift or stop codon mutations) or splicing site changes on both alleles (group 3). The comparison between these three groups showed that both the age at onset and ascertainment were significantly heterogeneous among them $(\mathrm{F}=6.43, P<0.01$ and $\mathrm{F}=13.37, P<0.005$, respectively). Comparison among the three groups showed that they were significantly higher in group 1 $(18.73 \pm 7.05$ for onset and 28.4 \pm 7.36 for ascertainment) than for both group $2(11.62 \pm 1.4$ for onset and $16.30 \pm 4.19$ for ascertainment) and 3 (10.57 \pm 3.25 for onset and $20.82 \pm 11.15$ for ascertainment). However, the mean ages of onset and ascertainment did not differ significantly between groups 2 and 3, as indicated by the Tukey-Kramer HSD test. The observation that patients who carry two null mutations are on average more severely affected than those who carry two missense mutations or one missense and one in-frame deletion is in accordance with Richard et al. ${ }^{16}$ However the fact that the mean ages of onset and ascertainment did not differ between groups 2 and 3 was unexpected and suggests that one null mutation is enough to determine a more severe phenotype.

\section{Serum creatine-kinase}

The mean increase in serum CK activity, at ascertainment, was significantly higher among males $(23.90 \pm 25.91)$ than among females $(13.89 \pm 12.21 ; P<0.05)$ as expected since on average serum CK is higher in males than in females. In addition, affected males were on average 2 years younger at ascertainment than females and it is known that serum CK activity decreases with the progression of the disease. ${ }^{31}$ Serum CK activity was also significantly higher among African-Brazilian than Caucasian patients $(\mathrm{F}=5.989 ; P=0.017)$ in accordance with our previous results in the normal Brazilian population. ${ }^{32}$ On the other hand the mean serum CK activity did not differ significantly according to the nature of the mutation between the 3 groups, probably because it is known to be highly variable during the course of the disease. ${ }^{1}$

\section{Clinical course in males as compared to females}

A greater proportion of affected males than females has been reported for other autosomal forms of muscular dystrophy, such as facioscapulohumeral muscular dystrophy (FSHD1). ${ }^{33,34}$ For FSHD1, this apparent difference among gender is due to the observation that the clinical picture is on average more severe in males than females and to a greater proportion of asymptomatic females carrying the deleterious FSHD1 mutation. ${ }^{34}$ On the other hand, for LGMD2A, Pollitt et al. ${ }^{35}$ found the inverse situation in the British population, that is, a slightly younger age at onset in female than in male patients, although the sample was relatively small (seven males and six females).
In the present sample, when we compared both sexes, it was observed that, the mean age at onset and ascertainment did not differ significantly between males $(12.12 \pm 3.46$ and $22.22 \pm 9.7$, respectively) and females $(16.56 \pm 9.73$ and $24.40 \pm 11.99$, respectively, $P>0.05)$. However, although the proportion of affected males (36) did not differ from the proportion of affected females (38), seven males were confined to a wheelchair (mean age $15.6 \pm 5.6$ ) while only two females were non-ambulant at age 40 and 45 . This observation suggests a more rapid progression in LGMD2A affected males than in females. Interestingly, in a recent screening of calpain-3 deficiency through muscle protein analysis in Italian patients ${ }^{9}$ there were significantly more affected males (43) than females (23). Although this gender disproportion was not discussed by the authors it called our attention since it is well known that more severely affected patients are more likely to be ascertained, in accordance to our suggestion.

\section{Effect of ethnic background}

The Brazilian population is highly miscigenated. Therefore, in order to analyse if the ethnic background could influence the severity of the clinical course, patients were classified in two groups: Caucasians (C) and African-Brazi$\operatorname{lian}(\mathrm{A} / \mathrm{B})$.

However, since null mutations were significantly more frequent among A/B than $\mathrm{C}$ patients (homozygous missense mutations were found only among Caucasians) any difference in the clinical course could represent a bias of ascertainment. In order to circumvent this possibility we compared patients with the same type of mutation. This was possible only for patients with null mutations in both alleles where the two groups ( $21 \mathrm{C}$ versus $17 \mathrm{~A} / \mathrm{B}$ ) were large enough to allow such a comparison. The mean age at onset and ascertainment was still lower among A/B (10.25 \pm 2.76 and $16.56 \pm 7.65$ respectively) than among $C$ patients $(11.12 \pm 4.19$ and $26.29 \pm 11.78$ respectively). However, the difference was statistically significant $(P<0.05)$ only for age at ascertainment, which occurred on average 10 years earlier in the A/B group, although there was a wide overlap. The fact that the difference was significant only for the age at ascertainment might be due to a more rapid progression in the latter group. Indeed, Pollitt et al. ${ }^{35}$ observed among CAPN3 British patients whose age at onset is not necessarily a predictor of the rate of progression. However, this parameter may be always interpreted with caution because many patients do not recall exactly when they noticed the first symptoms, particularly those with a lower cultural level.

In summary, the main findings of the present study are: (a) The identification of seven novel pathogenic changes and two recurrent mutations in the CAPN3 gene representing a founder effect, (b) The screening of 6 exons allowed the identification of $80 \%$ of the mutated alleles in the CAPN3 gene. This corresponds to approximately $24 \%$ of 
the adult forms of LGMD with important diagnostic applications, (c) Genotype:phenotype correlation showed that although highly variable, on average patients with two missense (one missense/one in frame deletion) mutations have a slower course than those with two nonsense mutations. However the phenotype in patients who carry two null mutations did not differ from those who carry just one null mutation, (d) The mean age of ascertainment in African/Brazilian occurs significantly earlier than among Caucasian LGMD2A patients carrying the same type of mutation suggesting a more severe course, (e) The finding of one patient with two identified mutations but with a borderline calpain-3 amount suggests that the proportion of calpainopathy based on muscle calpain-3 deficiency may be underestimated. Unclassified patients in whom other muscle protein showed normal results should be investigated for calpain mutations.

\section{Acknowledgements}

The collaboration of the following persons is gratefully acknowledged: Anita Kai, Rita Viegas, Tatiana Jazedje, Alessandra Splendore, Kikue Abe, Agnes Nishimura, Andrea Bernardino, Manuela Toninni, Carlos Maranduba, Eloisa de Sá Moreira for their scientific help and to Constância Urbani, Antônia Cerqueira and Marta Canovas for their secretarial and technical help. We would like to thank ABDIM and the affected families that collaborated with this research. This work was supported by FAPESP/CEPID, PRONEX, CNPq, IAEA. V Nigro is recipient of grants from Telethon (Italy) and MIUR (P.R.I.N.).

\section{References}

1 Zatz M, Vainzof M, Passos-Bueno MR: Limb-girdle muscular dystrophy: one gene with different phenotypes, one phenotype with different genes. Curr Opin Neurol 2000; 13: 511-517, (Review).

2 Bushby KM: The limb-girdle muscular dystrophies-multiple genes, multiple mechanisms. Hum Mol Genet 1999; 8: 1875 1882, (Review).

3 Hackman P, Richard I, Vihola A, Haravuori H, Marchand S, Labeit S, Sarparanta J, Taivainen A, Carpén O, Udd B: Tibial Muscular Dystrophy (TMD), 2q31 linked Myopathy: Sequencing and Functional Studies of the Titin (TTN) gene. Journal of the Neurological Sciences 2002; 199 (Suppl 1): S35.

4 Moreira ES, Wiltshire TJ, Faulkner G et al: Limb-girdle muscular dystrophy type $2 \mathrm{G}$ is caused by mutations in the gene encoding the sarcomeric protein telethonin. Nat Genet 2000; 24: 163-166.

5 Halliday W, Greenberg CR, Wrogemann K: Genetic heterogeneity of limb girdle muscular dystrophy in Manitoba Hutterites. Am J Hum Genet 1998; 639 (Suppl): A392, (Abstract 2280).

6 Passos-Bueno MR, Vainzof M, Moreira ES, Zatz M: The seven autosomal recessive limb-girdle muscular dystrophy (LGMD): from Igmd2a to Igmd2g. Am J Med Genet 1999; 82: 392-398.

7 Speer MC, Vance JM, Grubber JM et al: Identification of a new autosomal dominant limb-girdle muscular dystrophy locus on chromosome 7. Am J Hum Genet 1999; 64: 556-562.

8 Starling AL, Vainzof M, Pavanello R, Canovas M, Cerqueira A, Passos-Bueno MR, Zatz M: Evidence of further genetic heterogeneity for both autosomal dominant and autosomal recessive limb-girdle muscular dystrophy. Am J Hum Genet 2001; 69: 1942 (Suppl 1)
9 Fanin M, Pegoraro E, Matsuda-Asada C, Brown RH, Angelini C: Calpain-3 and dysferlin protein screening in patients with limbgirdle dystrophy and myopathy. Neurology 2001; 1: 660-665.

10 Beckmann JS, Richard I, Hillarie D et al: A gene for limb girdle muscular dystrophy maps to chromosome 15 by linkage. $C R$ Acad Sci Paris 1991; 132: 141-148.

11 Carafoli E, Molinari M: Calpain: a protease in search of a function?. Biochem Biophys Res Commun 1998; 247: 193-203, (Review 18).

12 Ono Y, Sorimachi H, Suzuki K: Structure and physiology of calpain, an enigmatic protease. Biochem Biophys Res Commun 1998; 245: 289-294, (Review 17).

13 Suzuki K, Sorimachi H: A novel aspect of calpain activation. FEBS Lett 1998; 433: 1-4, (Review 14).

14 Sorimachi H, Ono Y, Suzuki K: Skeletal muscle-specific calpain, p94, and connectin/titin: their physiological functions and relationship to limb-girdle muscular dystrophy type 2A. Adv Exp Med Biol 2000; 481: 383-395, (discussion); 395-397, (Review).

15 Baghdiguian S, Martin M, Richard I et al: Calpain 3 deficiency is associated with myonuclear apoptosis and profound perturbation of the IkappaB alpha/NF-kappaB pathway in limb-girdle muscular dystrophy type 2A. Nat Med 1999; 5: 503-511.

16 Richard I, Roudaut C, Saenz A et al: Calpainopathy-a survey of mutations and polymorphisms. Am J Hum Genet 1999; 64: $1524-1540$

17 Chae J, Minami N, Jin Y, Nakagawa M, Murayama K, Igarashi F, Nonaka I: Calpain 3 gene mutations: genetic and clinico-pathologic findings in limb-girdle muscular dystrophy. Neuromuscul Disord 2001; 11: 547-555.

18 Bushby KM, Beckmann JS: The limb-girdle muscular dystrophies-proposal for a new nomenclature. Neuromuscul Disord 1995; 5: $337-343$.

19 Azevedo ES: Subgroup studies of back admixture within a mixed population of Bahia, Brazil. Ann Hum Genet 1980; 44: 55-60.

20 Anderson LV, Davison K, Moss JA et al: Characterization of monoclonal antibodies to calpain 3 and protein expression in muscle from patients with limb-girdle muscular dystrophy type $2 \mathrm{~A}$. Am J Pathol 1998; 153: 1169-1179.

21 Vainzof M, Anderson LV, McNally EM et al: Dysferlin protein analysis in limb-girdle muscular dystrophies. J Mol Neurosci 2001; 17: 71-80.

22 Pogue R, Anderson LV, Pyle A et al: Strategy for mutation analysis in the autosomal recessive limb-girdle muscular dystrophies. Neuromuscul Disord 2001; 11: 80-87.

23 Miller SA, Dykes DD, Polesky HF: A Yesple salting out procedure for extracting DNA from human nucleated cells. Nucleic Acids Res 1988; 16: 1215.

24 Richard I, Broux O, Allamand V et al: Mutations in the proteolytic enzyme calpain 3 cause limb-girdle muscular dystrophy type $2 \mathrm{~A}$. Cell 1995; 81: $27-40$.

25 Allamand V, Broux O, Richard I et al: Preferential localization of the limb-girdle muscular dystrophy type $2 \mathrm{~A}$ gene in the proximal part of a 1-cM 15q15.1-q15.3 interval. Am J Hum Genet 1995; 56: $1417-1430$.

26 Weber JL, May PE: Abundant class of human DNA polymorphisms which can be typed using the polymerase chain reaction. Am J Hum Genet 1989; 44: 388-396.

27 Welch BL: On the comparison of several mean values: an alternative approach. Biometrika 1951; 38: 330-336.

28 Urtasun M, Saenz A, Roudaut C et al: Limb-girdle muscular dystrophy in Guipuzcoa (Basque Country, Spain). Brain 1998; 121: $1735-1747$

29 Fardeau M, Eymard B, Mignard C, Tome FM, Richard I, Beckmann JS: Chromosome 15-linked limb-girdle muscular dystrophy: clinical phenotypes in Reunion Island and French metropolitan communities. Neuromuscul Disord 1996; 6: 447-453.

30 Richard I, Brenguier L, Dincer P et al: Multiple independent molecular etiology for limb-girdle muscular dystrophy type 2A patients from various geographical origins. Am J Hum Genet 1997; 60: $1128-1138$. 
31 Zatz M, Vainzof M, Passos-Bueno MR (ed): Serum Creatine-Kinase (CK) In progressive Muscular Dystrophy; in Bushby KMD, Anderson LVB (ed). Totowa, New Jersey: Human Press, 2001, pp 31-49.

32 Passos-Bueno MR, Rabbi-Bortolini ER, Azevedo E, Zatz M: Racial effect on serum creatine-kinase for females at-risk for Duchenne dystrophy. Clin Chim Acta 1989; 179: 153-168.

33 van der Maarel SM, Deidda G, Lemmers RJLF et al: De novo facioscapulohumeral muscular dystrophy: Frequent somatic mosaicism, sex-dependent phenotype, and the role of mitotic transchromosomal repeat interaction between chromosomes 4 and 10. Am J Hum Genet 2000; 66: 26-35.
34 Zatz M, Marie SK, Cerqueira A, Vainzof M, Pavanello RC, PassosBueno MR: The facioscapulohumeral muscular dystrophy (FSHD1) gene affects males more severely and more frequently than females. Am J Med Genet 1998; 77: 155-161.

35 Pollitt C, Anderson LV, Pogue R, Davison K, Pyle A, Bushby KM: The phenotype of calpainopathy: diagnosis based on a multidisciplinary approach. Neuromuscul Disord 2001; 11: 287-296. 